\title{
Design of robust and energy efficient ATO speed profiles of metropolitan lines considering train load variations and delays
}

\author{
Adrián Fernández Rodríguez \\ IIT-ICAI, School of Engineering, Comillas Pontifical University, Madrid, Spain \\ E-mail: adrian.fernandez@iit.upcomillas.es
}

\begin{abstract}
Metropolitan railway operators' strategic plans include nowadays actions to reduce energy consumption. The application of ecodriving initiatives in lines equipped with ATO (Automatic Train Operation) systems can provide important savings with low investments.

Previous studies carried out under ATO framework have not considered the main uncertainties in the traffic operation: the train load and delays in the line. This paper proposes a method to design robust and efficient speed profiles to be programmed in the ATO equipment of a metro line. First, the optimal Pareto front of robust ATO speed profiles to changes in train load is generated for two objectives, running time and energy consumption. A robust optimization technique and an alternative method based on the conservation of the shape of the speed profiles (pattern robustness) are compared. Both procedures make use of MOPSO (Multi Objective Particle Swarm Optimization) algorithm.

Then, the set of speed profiles to be programmed in the ATO equipment is selected from the robust Pareto front by means of an optimization model. This model is a Particle Swarm Optimization algorithm (PSO) to minimise the total energy consumption considering the statistical information about delays in the line. This procedure has been applied to a case study. The results showed that the pattern-robustness is more restrictive and meaningful than the robust optimization technique as it provides information about shapes that are more comfortable for passengers. And the use of statistical information about delays by the proposed selection model provides additional energy savings between $3 \%$ and $14 \%$
\end{abstract}

Index Terms-Communication Based Train Control (CBTC), energy saving, multi objective particle swarm optimization (MOPSO), subway systems, train load variations, train operation, uncertainty.

\section{INTRODUCTION}

$\mathrm{M}$ ETROPOLITAN systems consume a huge amount of energy every day causing a large mass of CO2 emissions [1]. Furthermore, the energy prices are growing and the energy cost is becoming an important part in the cost structure of railway companies. As a consequence, new strategies are being implemented to reduce energy consumption. Some of these efficiency strategies are focused on improving the traffic management from the point of view of traffic design [2]-[6] and regulation [7]-[11], on the optimal use of regenerative braking [12]-[14] and on eco- driving [15]-[17]. These actions can be implemented in the short term with low investments compared with other energy efficiency actions as the renewal of trains [18] or the improvement of the infrastructure.

The eco-driving design calculates the speed profile with the minimum energy consumption for a target running time between stations. Typically, the techniques applied for this design are mathematical programming models and simulationbased models. Mathematical optimization models frequently need to simplify some aspects of train motion and energy calculation which make difficult the application to real cases [19]-[27]. On the other hand, simulation-based optimization algorithms are more flexible, do not require important simplifications, and the results are more accurate and practical. This accuracy is especially important in metropolitan lines equipped with Automatic Train Operation systems (ATO). Different optimization techniques have been used in combination with simulation - Genetic Algorithms (GA) [28]-[37], Artificial Neural Networks [38], [39], a combination of both techniques [40], Differential Evolution algorithms (DE) [41], Ant Colony Optimization (ACO) [31], prediction techniques [42] and direct search methods [43], [44].

Most of the modern metropolitan lines are equipped with ATO [45]. The onboard ATO equipment receives at stations the driving commands of a speed profile from the centralized regulation system. The regulation system on-line selects a speed profile from a pre-programmed set depending on the required arrival time. These pre-programmed speed profiles are characterized by running time, energy consumption and driving commands. Regulation system selects a speed profile with running time lower than or equal to the required running time. The application of the selected speed profile could produce a slight schedule advance. However, this schedule advance is easily compensated increasing a few seconds the dwell time at the next station.

The driving commands are usually made up by four parameters: coasting speed, remotoring speed, holding speed and braking rate [46]. In coasting remotoring cycles, when the train is tractioning and reaches the coasting speed, the traction is set to 0 to start coasting. Then, when the train reduces the speed up to the coasting speed minus the remotoring speed, the traction force is applied again. On the other hand, holding speed command produces a different kind of speed profile. This command forces the train to reach and maintain certain 
speed if it is possible. Braking rate defines the deceleration that the train will apply in the final braking. Every combination of the possible values of these parameters results in a particular speed profile with an associated running time and energy consumption between two stations.

In metropolitan lines equipped with Fixed Block signalling systems, the driving parameters are transmitted to the trains via encoded balises placed at stations, using a short number of bits due the bandwidth limitation of the balise. Due to the limited bandwidth of the Fixed Block system, the commands take discrete values between a maximum and a minimum with a big increment as shown in Table I [51]. Therefore, the number of different speed profiles that the ATO can execute is relatively small. This solution space can be exhaustively explored in order to find the speed profiles with minimum energy consumption for each running time (Pareto front). The ATO equipments are usually programmed with four alternative speed profiles (fast, regular, slow and extra-slow) for each station. The regulation system selects online the ATO pre-programmed speed profile with the closest running time to the target one (always below it) [46]. In [47], a method to design the ATO pre-programmed speed profiles in Fixed Block metro lines, based on detailed simulation, is presented. In the work of [47] the Pareto front is generated taking into account passenger comfort requirements. In addition, a procedure to select the four pre-programmed speed profiles based on heuristic rules is proposed.

TABLE I

VALUES OF THE ATO COMMANDS IN FIXED BLOCK SIGNALLING SYSTEMS [51]

\begin{tabular}{ccccc}
\hline \hline & $\begin{array}{c}\text { Braking rate } \\
\left(\mathrm{m} / \mathrm{s}^{2}\right)\end{array}$ & $\begin{array}{c}\text { Holding } \\
\text { speed }(\mathrm{km} / \mathrm{h})\end{array}$ & $\begin{array}{c}\text { Coasting } \\
\text { speed }(\mathrm{km} / \mathrm{h})\end{array}$ & $\begin{array}{c}\text { Re-motoring } \\
\text { speed }(\mathrm{km} / \mathrm{h})\end{array}$ \\
\hline Minimum & 0.6 & 30 & 30 & 10 \\
Maximum & 0.75 & 75 & 75 & 30 \\
Increment & 0.05 & 5 & 5 & 10 \\
\hline \hline
\end{tabular}

The new metropolitan signalling system Moving Block CBTC (Communication Based Train Control) [48], uses bidirectional wireless communications providing high bandwidth between the control centre and the trains. Therefore, the driving parameters can use practically continuous values. For this reason the solution space rises exponentially and makes impractical the use of exhaustive search to generate the optimal Pareto front. Several studies have analyzed the possible impact of CBTC system in energy efficiency. In [49], [50] new tracking control algorithms of two successive trains in CBTC system are proposed to reduce energy consumption. In [51] a detailed simulator is used in combination with the Multi Objective Particle Swarm Optimization (MOPSO) to obtain the Pareto curve of the possible speed profiles. An ACO max-min ant (MMA) algorithm is proposed in [52] on the framework of CBTC system, for the single objective problem.

The CBTC system also allows increasing easily the number of pre-programmed speed profiles with respect to the Fixed Block signalling systems. However, potential advantages of this increment have not yet been analyzed.

The previous studies focused on the design of ATO speed profiles do not take into account the principal sources of uncertainty in the traffic operation. These uncertainties are the train load and the train delays. The high precision of the ATO equipment in the execution of the pre-programmed driving parameters is practically just affected by the uncertainty in the mass of the train associated with the passenger load [53]. The CBTC system permits pre-programming different sets of speed profiles for different operation periods, as peak-hours and off-peak-hours. At each period, the passengers' load takes a different mean value, but it is not constant throughout the period because the passengers flow and the trains headway are not constant. In [51], the ATO speed profiles are optimized using a mean value of train mass in the period studied.

The other main source of uncertainty associated with the traffic operation is the occurrence of delays that must be corrected by the traffic regulator [53]. The statistical distribution of the delays determines the frequency with which the controller demand each pre-programmed ATO speed profile at each station. Typically, the four selected speed profiles of the pre-programmed set are equidistant in time, and the frequency of use is not taken into account, [47].

In this paper, a new method to design efficient and robust ATO speed profiles in CBTC lines is proposed, considering the previously described sources of uncertainty.

This method is composed by two steps. In the first step, the Pareto curve of robust ATO speed profiles, taking into account the uncertainty in the mass, is generated. Two procedures are applied to generate the robust Pareto curve. The first one is a robust optimization technique in time and energy consumption. An alternative procedure, based on the analysis of the relationship between the robustness and the conservation of the shape of the speed profiles (pattern robustness), is proposed. This procedure makes use of the MOPSO algorithm described in [51] adding a driving pattern identifier. In the second step, the set of ATO speed profiles to be programmed in the regulation system is selected from the robust Pareto front by means of an optimization model. This model is based on the Particle Swarm Optimization algorithm (PSO) [54] to minimize the energy consumption throughout an operation period, taking into account the statistical distribution of running times demanded by the regulation system.

In section II the two proposed methods to obtain a robust Pareto front of the speed profiles are presented. Section III describes the optimization model to select the preprogrammed speed profiles. The methodology proposed is applied to a case study and the results and analysis obtained from the design of ATO speed profiles are discussed in section IV. In addition, in section IV the energy savings associated with the increase of the number of ATO speed profiles to program in the regulation system are analyzed. Finally, conclusions are presented in section $\mathrm{V}$.

\section{DESIGN OF EFFICIENT AND ROBUST ATO SPEED PROFILES}

In this section, the Pareto curve of optimal ATO speed profiles is calculated, where the objectives are the running time and the energy consumption. For each running time the optimization algorithm has to find the ATO speed profile with 
the minimum consumption. For example, in Fig. 1, two speed profiles with the same running time are shown. These optimal speed profiles are later used by the regulation system in an operation period (peak or non-peak hour). The speed profiles must be robust against variations of the train mass in that period. The decision variables are the driving parameters of the speed profiles, that is, the typical configuration variables of the ATO equipment: coasting speed $\left(s_{c}\right)$, re-motoring speed $\left(s_{r}\right)$, holding speed $\left(s_{h}\right)$ and braking rate $(b)$. The ATO sets the train traction to zero (coasting) when train speed reaches the coasting speed value $s_{c}$, and the ATO sets train traction to maximum force when train speed gets down to $s_{c}$ minus the remotoring speed value $s_{r}$. When the train is controlled by a holding speed parameter $s_{h}$, the ATO regulates the train traction to maintain the train speed to this value. Each ATO speed profile (a possible solution) is defined as a configuration vector of driving parameters $\hat{x}=\left(s_{c}, s_{r}, s_{h}, b\right)$.

In the following subsections the robust optimization method is described and also the alternative procedure based on conservation of the driving pattern.

\section{A. Generation of robust Pareto front of optimal speed profiles using a robust optimization technique}

A robust solution is the less sensitive to perturbations in the decision variables in its vicinity [55]. The multi-objective optimization approach deals with the search of solutions which are non-dominated by any other feasible solutions. Thus, it is necessary to check the sensitivity of each solution $\hat{x}$ in the $M$ objectives to changes in all the decision variables to provide a robust Pareto front.

In [55], two approaches are proposed to obtain the robust optimal front. In order to obtain a multi-objective robust solution Type-I a mean effective objective function $\left(\hat{f}^{e f f}(\hat{x})\right)$ is used for the optimization instead of the original objective function $(\hat{f}(\hat{x}))$. Therefore, a solution $\hat{\mathrm{x}}^{*}$ is a robust solution of Type-I if it belongs to the Pareto-optimal solution of the following problem (1).

$$
\left.\begin{array}{c}
\text { Minimize } \hat{f}^{\text {eff }}(\hat{x})=\left(f_{1}^{\text {eff }}(\hat{x}), f_{2}^{\text {eff }}(\hat{x}), \ldots, f_{M}^{\text {eff }}(\hat{x})\right) \\
\text { subject to } \hat{x} \in S
\end{array}\right\}
$$

where $S$ is the solution space and $f_{j}^{e f f}(\hat{x})$ is the mean effective objective function of the $j$ objective for the neighborhood $\delta$. The mean effective objective function is defined in (2).

$$
f_{j}^{e f f}(\hat{x})=\frac{1}{\left|B_{\delta}\right|} \int_{\hat{y} \in \hat{x}+B_{\delta}} f_{j}(\hat{y}) d y
$$

where $\left|\mathrm{B}_{\delta}\right|$ is the hypervolume [55] of the chosen vicinity $\delta$.

The second approach proposed by Deb and Gupta adds a restriction to the original problem so that the user can adjust the maximum value of the solutions sensitivity. Therefore, a solution $\hat{x}^{*}$ is a robust solution of Type-II if it belongs to the Pareto-optimal solution of the problem (3):

$$
\left.\begin{array}{c}
\text { Minimize }\left(f_{1}(\hat{x}), f_{2}(\hat{x}), \ldots, f_{M}(\hat{x})\right) \\
\text { subject to } \frac{\left\|\hat{f}^{P}(\hat{x})-\hat{f}(\hat{x})\right\|}{\|\hat{f}(\hat{x})\|} \leq \eta \\
\hat{x} \in S
\end{array}\right\}
$$

where $\hat{f}^{P}(\hat{x})$ is the perturbed objective vector and $\eta$ is the maximum value of sensitivity required. The perturbed objective vector can be chosen either as the worst case or as the mean effective of the neighbourhood.

Type-II was chosen to obtain the optimal speed profiles because it is more practical than Type-I as it is highlighted in [55]. Furthermore, in the present ATO design problem, the worst cases, that is, the maximum variations of running time and energy consumption occur in the extreme values of train mass for the considered operation period. As previously mentioned, the high precision of the ATO equipment in the execution of the driving parameters is affected, in practice, only by the uncertainty in the mass of the train associated with the passenger load.

Therefore the Type-II robust optimization model has been selected because the robustness can be controlled by a maximum sensitivity value. The sensitivity is controlled comparing the objective function for the mean value of the train mass in the operation period with the worst case (maximum and minimum mass). The optimization problem is formulated as (4).

$$
\begin{aligned}
\text { Minimize } \hat{f}(\hat{x})=\left(f_{1}(\hat{x}), f_{2}(\hat{x}), \ldots, f_{M}(\hat{x})\right) \\
\text { subject to } \frac{\left\|\hat{f}_{\text {max }}^{P}(\hat{x})-\hat{f}(\hat{x})\right\|}{\|\hat{f}(\hat{x})\|} \leq \eta \\
\frac{\left\|\hat{f}_{\min }^{P}(\hat{x})-\hat{f}(\hat{x})\right\|}{\|\hat{f}(\hat{x})\|} \leq \eta \\
\hat{x} \in S
\end{aligned}
$$

where $\hat{f}_{\max }^{P}(\hat{x})$ and $\hat{f}_{\min }^{P}(\hat{x})$ are the objective vector for the maximum and the minimum mass, for the considered period, and $\hat{f}(\hat{x})$ is the objective vector for the mean value of the mass in that period.

A multi Objective Particle Swarm Optimization algorithm with crowding distance mechanism (MOPSO-CD) [51] is applied to generate the Pareto curve of the problem defined in (4).

In the MOPSO algorithm, each particle (possible solution) is treated as a point in an M-dimensional space. The particle "flies" over the search space to find promising regions of the landscape. The movement of a particle is directed by the best position found by itself (pbest) and the best position (gbest) found by the whole swarm (population). So the position and the velocity of the particle $i$ are updated at each iteration using (5) and (6).

$$
\begin{aligned}
& \hat{v}_{i}(n)=w \cdot \hat{v}_{i}(n-1)+c_{1} \cdot r_{1}\left(\hat{p}_{i}-\hat{x}_{i}(n-1)\right) \\
& +c_{2} \cdot r_{2}\left(\hat{p}_{g}-\hat{x}_{i}(n-1)\right)
\end{aligned}
$$

where $\hat{x}_{i}(\mathrm{n})$ and $\hat{v}_{i}(\mathrm{n})$ are the position and the speed of particle $i$ at iteration $n$. The positions $\hat{p}_{i}$ and $\hat{p}_{g}$ are respectively the pbest and the gbest. $c_{1}$ and $c_{2}$ are positive constants called ("social factors"), and $r_{1}$ and $r_{2}$ are random numbers between 0 and $1 . w$ is an inertia weight that controls the effect of the previous history of velocities.

The MOPSO algorithm deals with a multi-objective optimization problem where the objective is to find the Pareto 
curve. Pareto curve is the set of the non-dominated solutions, i.e., the solutions that cannot be improved in both energy consumption and running time. The objective function is shown in (7).

Minimize $\hat{f}(\hat{x})=\left(f_{1}(\hat{x}), f_{2}(\hat{x})\right)$

where $f_{1}(\hat{x})$ is the running time and $f_{2}(\hat{x})$ is the energy consumption.

The algorithm maintains an external archive $A$ that stores the non-dominated solutions found throughout the iterations. The gbest is randomly selected from the solutions in the archive $A$. However, if the solutions of $A$ were selected based on uniform probability distribution, the resulting Pareto curve would contain substantial gaps. To avoid this problem, the crowding distance MOPSO [56] selects the gbest from a top portion of the archive $A$ sorted in decreasing values of crowding distance (CD). The CD is calculated as the sum of distances in all the objectives of its two neighboring solutions

On the other hand, the pbest of a particle is selected as the current position if it dominates the previous pbest. If the previous pbest dominates the current position the pbest remains. However if the current position of the particle and its pbest are mutually non-dominated, the pbest is selected randomly between them.

\section{B. Alternative method to generate a robust Pareto front based on driving patterns}

The driving pattern determines the shape of the speed profile. A pattern is characterized by the sequence of the different working modes that the train executes during its journey. These working modes are traction (T), braking (B), coasting (C) and final braking (FB). Alternative solutions $\hat{x}$ are obtained exploring the variable decision space (values for $\left.s_{c}, s_{r}, s_{h}, b\right)$. They are simulated to calculate their associated pattern, running time and energy consumption.

In Fig. 1, two examples of driving patterns are presented. The ATO command is the ratio between the force demanded by the automatic driver and the maximum force that the motor can provide. Positive values of the ATO command produce traction force and negative values of the ATO command produce braking force. In coasting-remotoring stages the ATO command present typically two values (0 and 1 in Fig. 1). Zero value of the ATO command corresponds to a coasting phase (see [47] for more details about ATO model).

The driving pattern of a speed profile provides information to the designer about the passengers' comfort. Furthermore, information about the robustness of the speed profile can be obtained from the driving pattern. Frequently, the speed profiles which change their driving pattern with the train load have associated high variations in running time and energy consumption. A solution $\hat{x}$ is said to be pattern robust if its associated pattern does not change with train mass variations.

Therefore, the following alternative methodology is proposed to obtain a robust Pareto front based on the detection of changes in the pattern:

- Step 1: Obtain the Pareto front considering the mean value of the train mass in the operation period using the MOPSO-
CD algorithm without any robustness restriction

- Step 2: Identify the different driving patterns of the solutions obtained in step 1.

- Step 3: Eliminate the solutions with uncomfortable patterns according to the designer's criteria.

- Step 4: For each pattern p (not eliminated in step 3), obtain a different pattern-robust Pareto front using the MOPSO-CD adding the following restriction:

- $\quad$ The solution $\hat{x}$ must perform a pattern $\mathrm{p}$ using the mean value of the train mass (if not, $\hat{x}$ is discarded).

- $\quad$ The solution $\hat{x}$ must be pattern-robust for the worstcase train mass variations, that is, it must conserve the pattern $p$ simulating with the maximum and the minimum values of train mass in the operation period.

- Step 5: The global robust Pareto front is made by the nondominated solutions combining all the previous Pareto fronts of each $p$ in step 4 .

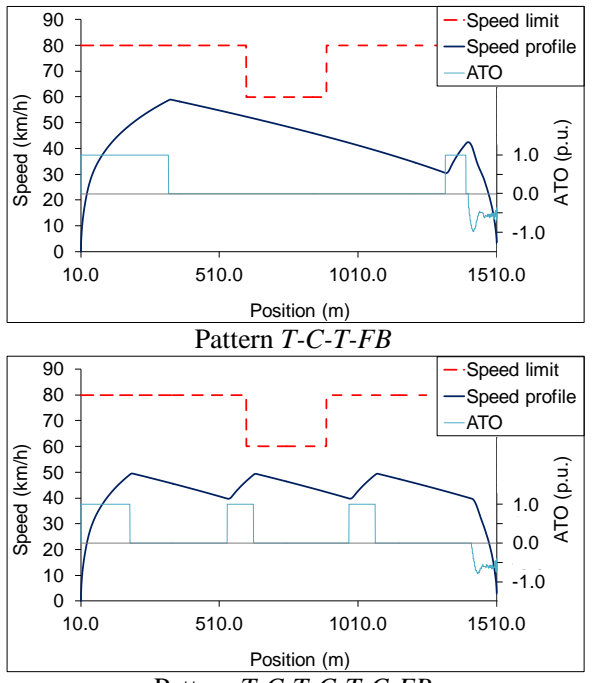

Pattern $T-C-T-C-T-C-F B$

Fig. 1: Example of different driving patterns

With this alternative method, the designer can filter or give priority to certain driving patterns over other patterns based on comfort criteria defined by the operator (typically the maximum number of coasting-remotoring cycles, and the minimum duration of the last traction before braking) [51]. In the case study explained in Section IV the solutions obtained using this method and using the method explained in Section II-A will be compared.

\section{OPTIMAL SELECTION OF THE ATO SPEED PROFILE SET}

Once the robust Pareto front has been obtained, the objective of the optimization method described in this section is to select a set of ATO speed profiles to be programmed in the ATO equipment. These speed profiles are optimized for a specific operation period (i.e. peak-hour or non-peak-hour) to be handled by the traffic regulation system during that period.

The candidates to be programmed are the speed profiles at the robust Pareto front obtained applying the method described in the previous section. Therefore the candidates are 
the most efficient speed profiles for each possible running time.

The problem is stated as a stochastic optimization model. The statistical distribution of running times demanded by the traffic regulation system between two stations in the considered operation period is an input data for the proposed model. Fig. 2 shows an example of a running time distribution, where $p_{s}$ is the statistical probability of demanding a running time. This figure shows a typical case where the highest probability is concentrated around the times close to the nominal running time (125 seconds). The scenarios where the regulation system demands higher running times than the nominal one occur when the train is slowed down by the regulation system to reduce its time interval with the following train [46]. On the other hand, scenarios that require lower running times than the nominal one occur when the train is delayed.

The statistical distribution of running times is discretized into NS scenarios with regular time intervals assigning a probability $p_{s}$ to each scenario of running time $T_{s}$ as shown in Fig. 2.

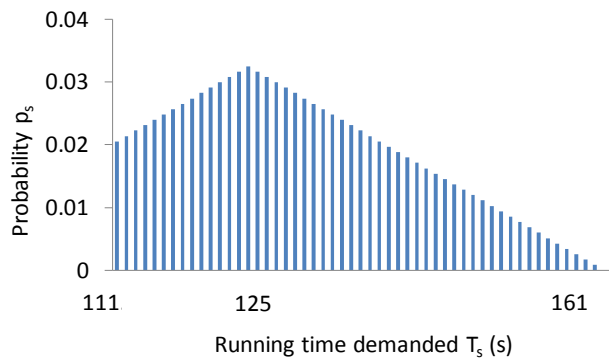

Fig. 2: Example of the discrete probability distribution of the running time demanded by the regulator

The objective is to select a set of $D$ number of speed profiles from the Pareto front (8).

$s t^{d} \in J \quad d=(1,2 . . \mathrm{D})$

where $s t^{d}$ is the $d$ speed profile of the pre-programmed set $\widehat{s t}$ with an associated running time $T_{d}$ and energy consumption $C_{d} . J$ is the Pareto front.

The energy consumption associated with the scenario $s$ is the energy consumption of the speed profile selected from the pre-programmed set by the traffic regulation system. In order to obtain the energy consumption of each scenario it is necessary to apply the selection logic of the regulation system (9).

$C_{s}=\left\{\min \left(C_{d}\right) \mid T_{d} \leq T_{s}\right\}$

The regulation system on-line selects the speed profile that has the lowest energy consumption from those that perform a running time lower than or equal to the demanded running time (9). The running time demanded by the traffic regulation system is constrained by the shortest running time associated with the interstation. Furthermore, the flat out speed profile will always be selected as an element of the pre-programmed set to ensure that there is a solution that fulfills (9) when the regulator demands the shortest time (see Figure 12 in the case study).

The aim of the problem is the selection of a set of speed profiles with the minimum expected energy consumption. Therefore the objective function is expressed in (10):

$\min \sum_{s}^{N S} p_{s} C_{s}$

Particle Swarm Optimization algorithm (PSO) [54], [57], [58] is used to solve this problem because its ability to work with discrete exploration spaces, its simplicity in concept and coding implementation and its less sensitivity to the nature of the objective function. The algorithm makes use of a swarm of $N P$ particles whose position $\left(\widehat{s t}_{i}\right)$ and velocity $\left(\hat{v}_{i}\right)$ is randomly initialized. The position $\widehat{s t}_{i}$ of the particle $i$ is defined by the speed profiles of the Pareto front $J$ selected to form the preprogrammed set $\widehat{s t}_{i}$. The information of the Pareto front is introduced in the algorithm assigning to each speed profile, besides their running time and energy consumption, an integer number $j$ which represents their position in the Pareto front respect to the other speed profiles when the Pareto set is time sorted. Therefore, the pre-programmed set $\widehat{s t}_{i}$ is a vector of $D$ dimensions where each dimension $s t_{i}{ }^{d}$ has a value $j$ assigned which represents the Pareto position of a speed profile selected in the pre-programmed set $\widehat{s t}_{i}$. The dimensions of each $\widehat{s t}_{i}$ vector are sorted as a function of the position of the Pareto position $j$ in order to increase the computational efficiency of the algorithm. The movement of the particles varies depending on their velocity which is updated at each iteration $n$. The value of the velocity depends on the best position found by the particle $\left(\hat{p}_{i}\right)$ and the best position found by the whole swarm $\left(\hat{p}_{g}\right)$ following (11) and (12).

$$
\begin{aligned}
& \hat{v}_{i}(n)=w \cdot \hat{v}_{i}(n-1)+c_{1} \cdot r_{1}\left(\hat{p}_{i}-\widehat{s t}_{i}(n-1)\right) \\
& +c_{2} \cdot r_{2}\left(\hat{p}_{g}-\widehat{s t}_{i}(n-1)\right) \\
& \widehat{s t}_{i}(n)=\widehat{s t}_{i}(n-1)+\hat{v}_{i}(n)
\end{aligned}
$$

where $c_{1}$ and $c_{2}$ are the "social factors" constants, $r_{1}$ and $r_{2}$ are random numbers between 0 and 1 and $w$ is an inertia weight that controls the effect of the previous history of velocities.

The pbest $\left(\hat{p}_{i}\right)$ and gbest $\left(\hat{p}_{g}\right)$ are obtained assigning at each solution a fitness value. The fitness value is the expected value of the energy consumption of the pre-programmed set. Thus, a lower value of fitness implies a better solution.

PSO algorithm is a single-objective algorithm. Therefore, it does not require an external archive $A$ because the final solution is the gbest in the last iteration (Fig. 3 and Fig. 4).

\section{CASE STUDY}

The methods previously described have been applied to a case study considering a typical journey between stations in Metro de Madrid of 1500 meters, with different speed limits along the journey (see Fig. 6). A class-3000 train of Metro de Madrid has been modeled. The empty mass of the train is 160 tons, the maximum passenger's load is 78 tons and the length of the train is 90 meters. The maximum power of the train is $1500 \mathrm{~kW}$ and the traction network is $1500 \mathrm{~V} \mathrm{DC}$. 
The speed profile design is carried out for an operation period characterized by an average passenger load of $50 \%$ of the maximum. Furthermore in the $90 \%$ of the situations the passenger load is between $30 \%$ and $70 \%$ of the maximum. These limits will be used to check the robustness conditions of the speed profiles to mass variations.

\begin{tabular}{|c|}
\hline 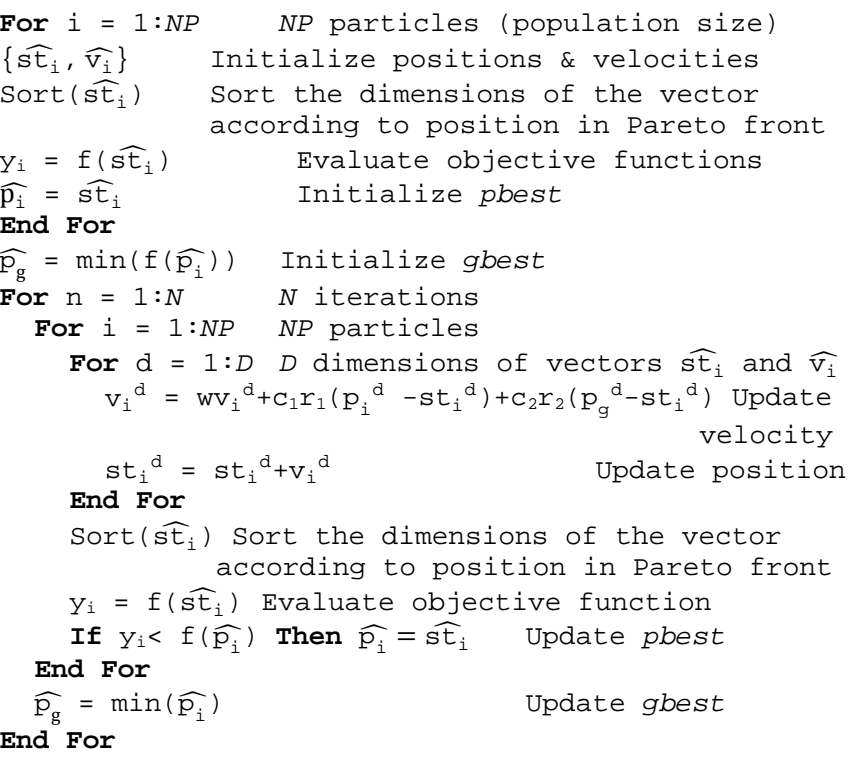 \\
\hline
\end{tabular}

Fig. 3: Pseudocode of PSO algorithm to obtain the energy efficient preprogrammed set

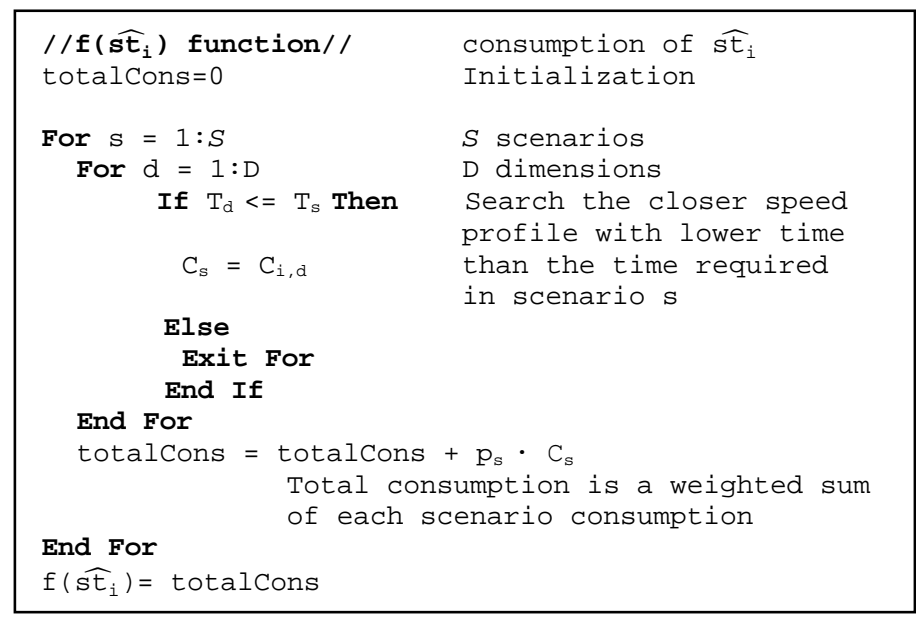

Fig. 4: Pseudocode of the algorithm used to calculate the expected value of the energy consumption of a solution

A detailed train simulator (average accuracy of $4.2 \%$ in traction energy and $1.0 \%$ in running times) and a simulator of real ATO equipment both described in [47] are used to calculate the running time and the energy consumption of speed profiles.

\section{A. Generation of the robust Pareto front}

The robust MOPSO-CD algorithm (Type-II) has been applied to the case study and the results obtained with the MOPSO-CD algorithm [51] and the proposed robust MOPSO$\mathrm{CD}$ are shown in Fig. 5. The robustness coefficient $\eta$ is set to
0.07 because this value has demonstrated an acceptable performance in the context of the problem.

Comparing both Pareto fronts, the robust one presents a wider time-gap around the running time $120 \mathrm{~s}$, and higher energy consumption from 135 to $145 \mathrm{~s}$.

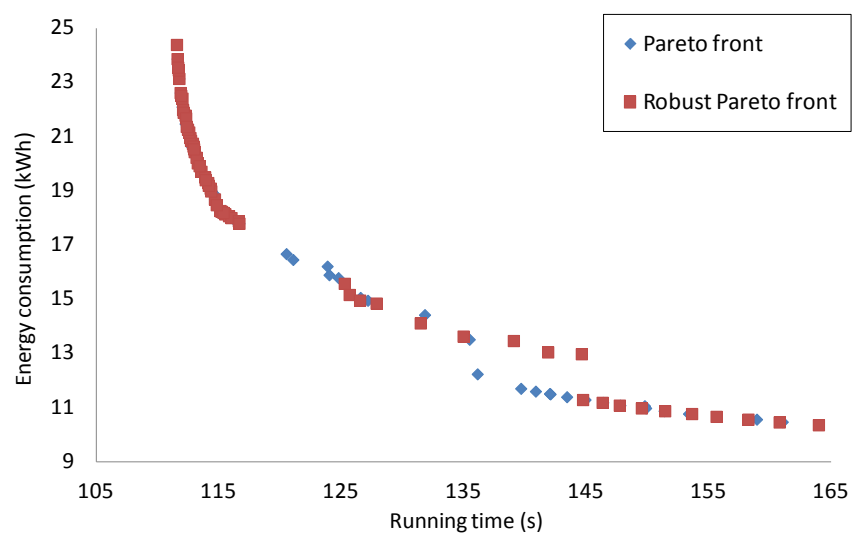

Fig. 5: Comparison of the current Pareto front and robust Pareto front using the robust MOPSO-CD algorithm

Non-robust solutions, that suffer great variations in time and energy consumption with train load, were analyzed. It was observed that most of these solutions change the shape of their driving pattern for extreme values of train load with respect to the average value. For instance, Fig. 6 shows two different speed profiles considering the same ATO parameters $\left(s_{c}=58\right.$ $\mathrm{km} / \mathrm{h}, s_{\mathrm{r}}=21 \mathrm{~km} / \mathrm{h}, b=0.65 \mathrm{~m} / \mathrm{s}^{2}$ ) but different passenger load (50\% and 100\%). The increase in the train mass causes a lower starting acceleration. For this reason, the train does not reach the coasting speed, and instead the control system activates the braking mode to fulfill the speed limitation of 60 $\mathrm{km} / \mathrm{h}$. This fact changes the shape of the speed profile and causes large differences in energy consumption and running time as shown in Table II.
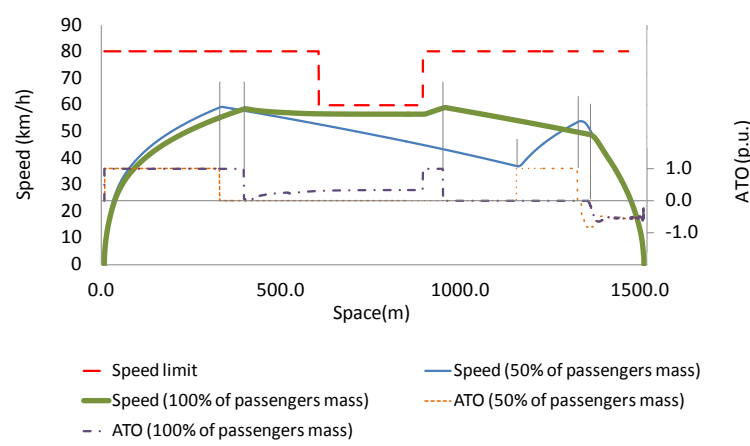

Fig. 6: Simulation of ATO configuration: $\left(s_{c}=58 \mathrm{~km} / \mathrm{h}, s_{\mathrm{r}}=21 \mathrm{~km} / \mathrm{h}, b=0.65\right.$ $\mathrm{m} / \mathrm{s}^{2}$ ) with $50 \%$ and $100 \%$ passenger load

This effect has been studied during the execution of the robust MOPSO-CD algorithm. With this purpose, pattern variations has been checked, and the number of pattern changes are counted separately for robust a non robust solutions. In addition, different values of the $\eta$ parameter have been considered in order to compare the results for higher and lower sensitivity (see Table III).

For $\eta$ higher than or equal to 0.07 , all the solutions rejected 
by the robust restriction change their pattern, so they are also non pattern robust to train load changes. On the other hand, there are many solutions that fulfill the robust restriction but change their pattern.

TABLE II

PERFORMANCE OF ATO CONFIGURATION: $\left(\mathrm{S}_{\mathrm{c}}=58 \mathrm{~km} / \mathrm{h}, \mathrm{S}_{\mathrm{r}}=21 \mathrm{~km} / \mathrm{h}, b=0.65 \mathrm{~m} / \mathrm{s}^{2}\right)$

\begin{tabular}{cccc}
\hline \hline Passengers' load & $\begin{array}{c}\text { Time } \\
(\text { seconds })\end{array}$ & $\begin{array}{c}\text { Energy consumption } \\
(\mathrm{kWh})\end{array}$ & Pattern \\
\hline $50 \%$ & 130.30 & 17.11 & T-C-T-FB \\
$100 \%$ & 121.25 & 20.73 & T-B-T-C-FB \\
\hline \hline
\end{tabular}

TABLE III

PERFORMANCE OF THE ROBUSTNESS RESTRICTION IN THE ROBUST MOPSO ALGORITHM FOR DIFFERENT $\eta$ VALUES

\begin{tabular}{cccc}
\hline \hline$\eta$ & $\begin{array}{c}\text { Rejected } \\
\text { solutions }\end{array}$ & $\begin{array}{c}\text { Rejected non pattern- } \\
\text { robust solutions }\end{array}$ & $\begin{array}{c}\text { Accepted non pattern- } \\
\text { robust solutions }\end{array}$ \\
\hline 0.15 & 78 & 78 & 585 \\
0.1 & 108 & 108 & 559 \\
0.07 & 132 & 132 & 482 \\
0.04 & 1206 & 568 & 374 \\
0.02 & 1275 & 322 & 115 \\
0.01 & 3948 & 3402 & 0 \\
\hline \hline
\end{tabular}

For $\eta$ values lower than 0.07 the situation changes: the robust algorithm rejects many solutions that are pattern robust, and a considerable amount of non pattern-robust solutions continue being accepted by the robust restrictions.

In conclusion, for high enough $\eta$, solutions that preserve the driving pattern against load changes are also robust in energy and time. This robustness criterion based on patterns is more restrictive and can be useful for designers, because it guarantees a qualitative level of passenger's comfort associated with the pattern (see next section).

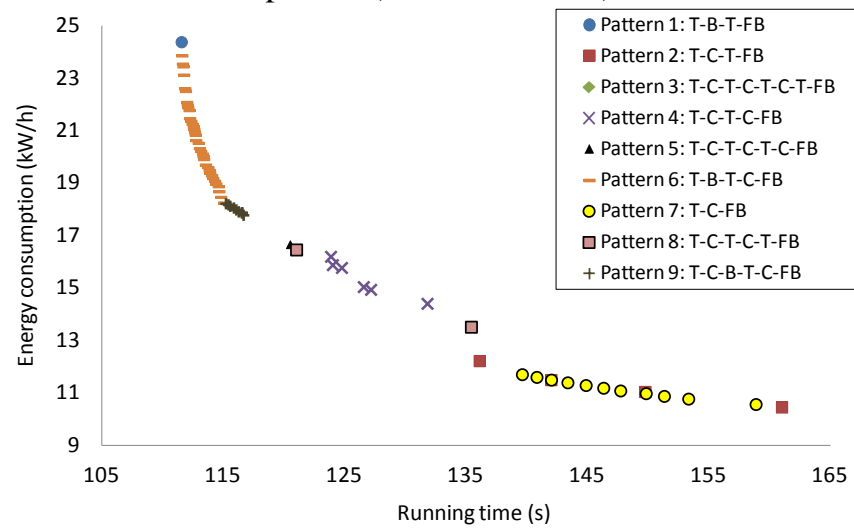

Fig. 7: Optimal Pareto front of the possible speed profiles and driving patterns obtained

\section{B. Generation of the robust Pareto front using the method based on driving pattern}

The first step to obtain the robust Pareto front based on maintaining the driving patterns is the identification of patterns present in the original Pareto front. For this purpose, the MOPSO algorithm without any robustness restriction and average load is executed to obtain the optimal front as shown in Fig. 7.

In this case study, there are 9 different driving patterns. The shape of some driving patterns is shown in Fig. 8. Patterns 3 and 8 are rejected because they present a short traction period before starting the final braking. This situation can be perceived by the passengers as uncomfortable.

For each non-filtered driving pattern, a Pareto front of the solutions that performs each pattern is obtained. A pattern restriction is added to the MOPSO algorithm to select solutions of the specific pattern. Then, the pattern-robustness of each solution is checked. All the solutions are simulated using the maximum value of the train load (70\%), and using the minimum one (30\%). Solutions that change the pattern (not pattern-robust) are eliminated and the result is a set of robust Pareto fronts for each driving pattern (Fig. 9).
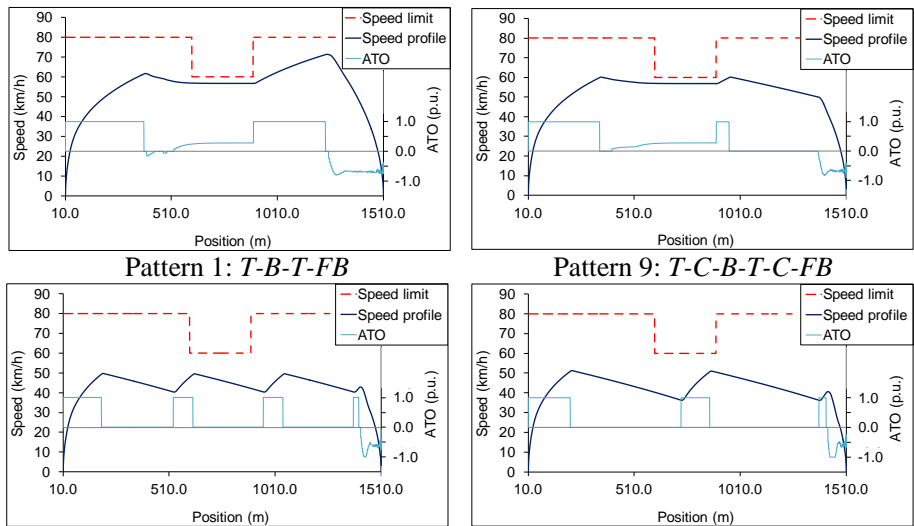

Pattern 3: T-C-T-C-T-C-T-FB

Pattern 8: T-C-T-C-T-C-FB

Fig. 8: Example of the speed profile of different patterns

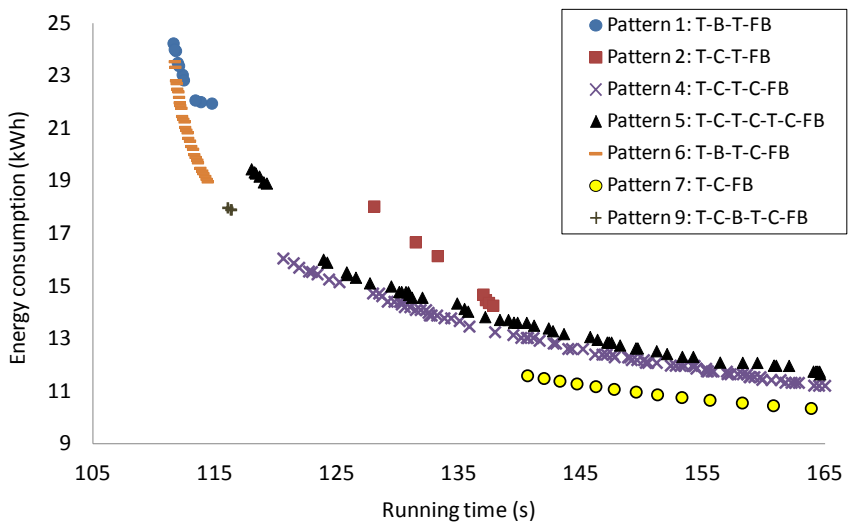

Fig. 9: Pattern-robust Pareto fronts for each driving pattern

Dominated solutions are eliminated to obtain a single robust Pareto front (Fig. 10).

It has been necessary to simulate an average of 5000 journeys to obtain each robust Pareto front.

In Fig. 11, the comparison of this solution with the previously obtained by using the robust MOPSO-CD algorithm for $\eta=0.07$, shows that the number of solutions is higher, and the running-time gaps have been reduced. The designer can select a speed profile taking into account not only its energy consumption but also the preferred driving patterns, considering the passenger comfort or other operation criteria.

\section{Optimal selection of the pre-programmed set of ATO speed profiles}

Traffic regulation systems typically handle a set of 4 preprogrammed alternative speed profiles between stations [46]. The first speed profile (number 0) performs the lowest running 
time (flat-out), and it is used to recover delays. The second speed profile (number 1 ) is usually chosen to provide the nominal running time. The third (number 2) and the fourth (number 3) speed profiles have higher associated running times and lower energy consumption, and are used to reduce the time interval with the following train. Typically, the speed profiles have been selected equidistant, that is uniformly distributed with the same time separation [47]

The optimization method explained in Section III has been applied to our case study to select the 4 speed profiles. The inputs of the proposed PSO algorithm are the robust Pareto front obtained in Section IV-B, the size of the preprogrammed set ( 4 speed profiles in this case study) and the discrete statistical distribution of running times demanded by the traffic regulation system at the control centre. Table IV shows the tuned parameters of the PSO algorithm.

\section{TABLE IV}

TUNED PARAMETERS OF THE PSO ALGORITHM

\begin{tabular}{ccccc}
\hline \hline $\mathrm{c}_{1}$ & $\mathrm{C}_{2}$ & $\mathrm{~W}$ & $\mathrm{NP}$ & Iterations \\
\hline 1 & 1 & 0.6 & 300 & 400 \\
\hline \hline
\end{tabular}

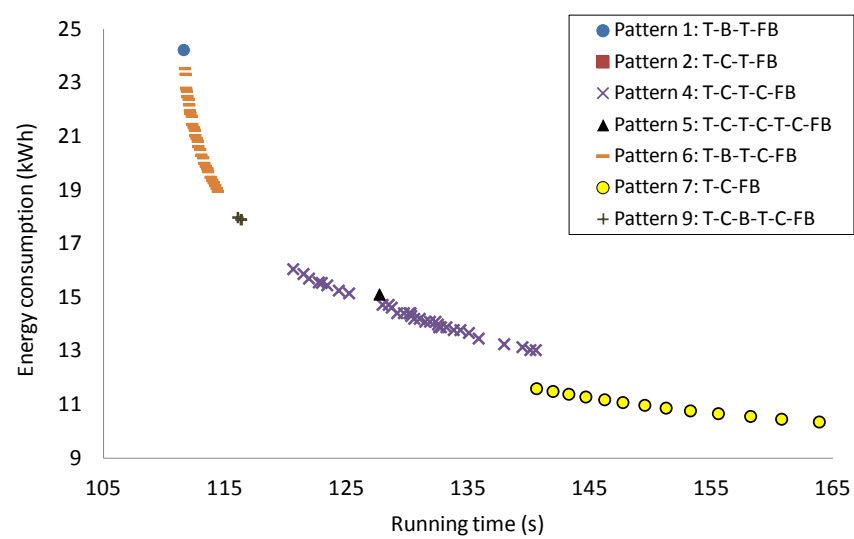

Fig. 10: Robust Pareto front obtained using the method based on driving pattern robustness

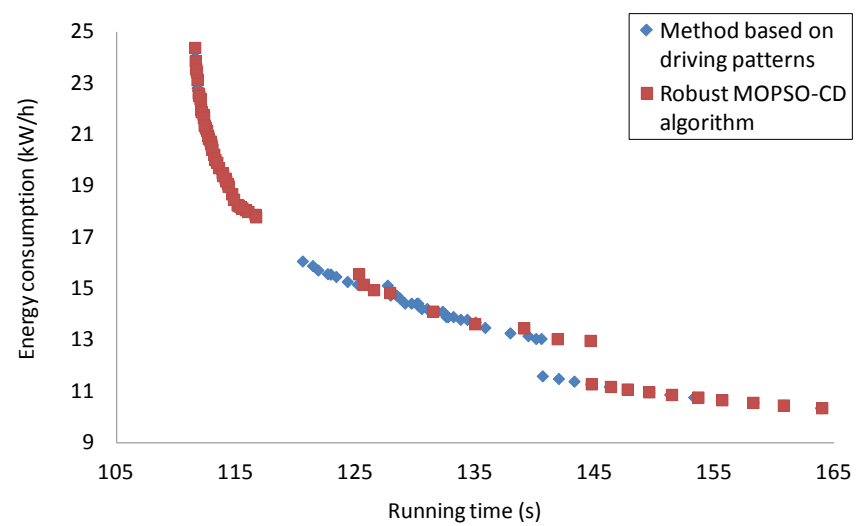

Fig. 11: Comparison between robust MOPSO-CD algorithm and the pattern robust algorithm

Three different shapes of probability distributions of demanded running times are considered in this study: decreasing, increasing and uniform distributions. The first one represents stations where delays are frequent, or the nominal running time is close to the minimum one. The second distribution represents stations where trains are regulated frequently to reduce their time interval with the following train, or the nominal running time is close to the maximum one. The third distribution corresponds to an intermediate situation where running times are demanded with the same probability.

Considering the previous distributions of demanded running times, the four optimal speed profiles are obtained by the proposed PSO algorithm (see Fig. 12).

Fig. 12 shows that the selected optimal solutions are not uniformly distributed in time (as the typical design criterion does), when the objective function is the minimization of the energy consumption along an operation period.

The PSO algorithm selects the speed profiles where the energy benefit is higher taking into account the frequency of use of the speed profiles. Fig. 12 shows that the optimal selection is the same for the decreasing and the uniform distributions, and the optimal selection is different in the increasing probability distribution case.

Table $\mathrm{V}$ shows the numerical results in terms of energy consumption and savings.

The speed profiles selected by the uniform distribution of running times are not the most efficient. The optimal selection of speed profiles generated by the PSO algorithm provides energy savings between $3.5 \%$ and $14 \%$ compared to the typical design criterion. These are additional savings to those obtained from the optimal design of the ATO speed profiles (that are around 20\%) described in [47]

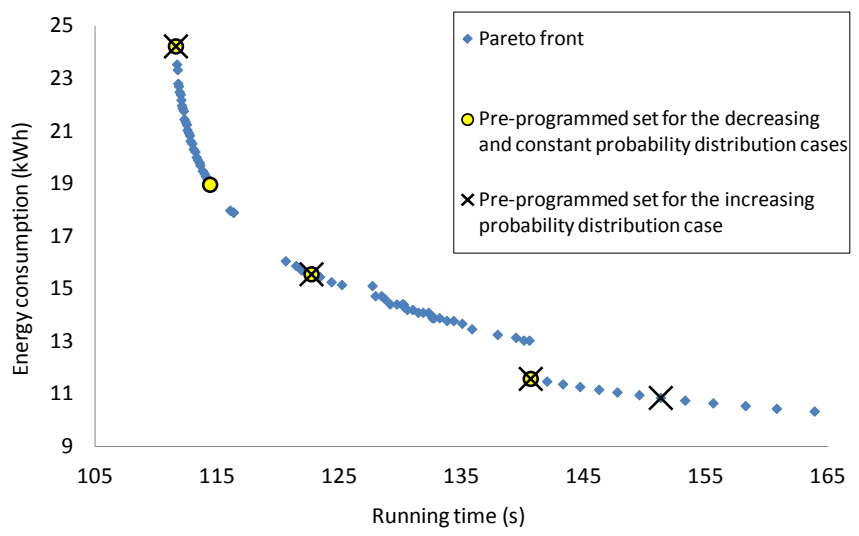

Fig. 12: Optimal speed profiles selected by the PSO algorithm

TABLE V

ENERGY CONSUMPTION AND SAVINGS OBTAINED COMPARING THE OPTIMIZATION RESULTS WITH THE EQUIDISTANTLY PRE-PROGRAMMED SET

\begin{tabular}{c|ccc}
\hline \hline & $\begin{array}{c}\text { Decreasing } \\
\text { prob. }(\mathrm{kWh})\end{array}$ & $\begin{array}{c}\text { Rising probability } \\
\text { distribution }(\mathrm{kWh})\end{array}$ & $\begin{array}{c}\text { Constant prob. } \\
(\mathrm{kWh})\end{array}$ \\
\hline Optimal & 16.64 & 12.90 & 14.78 \\
Equidistant & 17.25 & 15.02 & 16.11 \\
Savings & $3.5 \%$ & $14.1 \%$ & $8.3 \%$ \\
\hline \hline
\end{tabular}

Optimal solutions provide average advance time of 4,8s (decreasing and constant probability distribution case) and 6,5 s (increasing probability distribution case). These advance times are compensated increasing the dwell time, and thus, there is no schedule advance at departures.

In the previous optimization model, the number of speed profiles is a fixed parameter. However, the new signalling 
system based on radio communication, CBTC, allows the programming of more speed profiles in the equipments. In order to exploit this advantage, the energy efficiency that can be obtained increasing the number of speed profiles to be preprogrammed has been analyzed. In addition, when the number of speed profiles is increased, the schedule advance at arrivals is reduced.

The PSO algorithm has been executed with different sizes (from 2 to 20 speed profiles) of the pre-programmed set, taking into account the 3 previous probability distributions. The curves plotted in Fig. 13 represent the energy savings obtained for each size compared with size $=1$.

In view of the results, it can be concluded that the lower importance have the delays in the line the larger the achievable energy saving. The usual 4 speed profile preprogrammed set is in general an acceptable solution. Nevertheless, significant energy savings can be reached with 1 or 2 extra-speed profiles. Table VI compares the optimal sets of 4 and 6 speed profiles.

TABLE VI

COMPARISON OF THE OPTIMAL PRE-PROGRAMMED SET OF 4 AND 6 SPEED PROFILES

\begin{tabular}{c|ccc}
\hline \hline & $\begin{array}{c}\text { Decreasing } \\
\text { prob. }(\mathrm{kWh})\end{array}$ & $\begin{array}{c}\text { Increasing prob. } \\
(\mathrm{kWh})\end{array}$ & $\begin{array}{c}\text { Constant prob. } \\
(\mathrm{kWh})\end{array}$ \\
\hline 6 speed profiles & 16.04 & 12.43 & 14.31 \\
4 speed profiles & 16.64 & 12.90 & 14.78 \\
Savings & $3.64 \%$ & $3.60 \%$ & $3.19 \%$ \\
\hline \hline
\end{tabular}

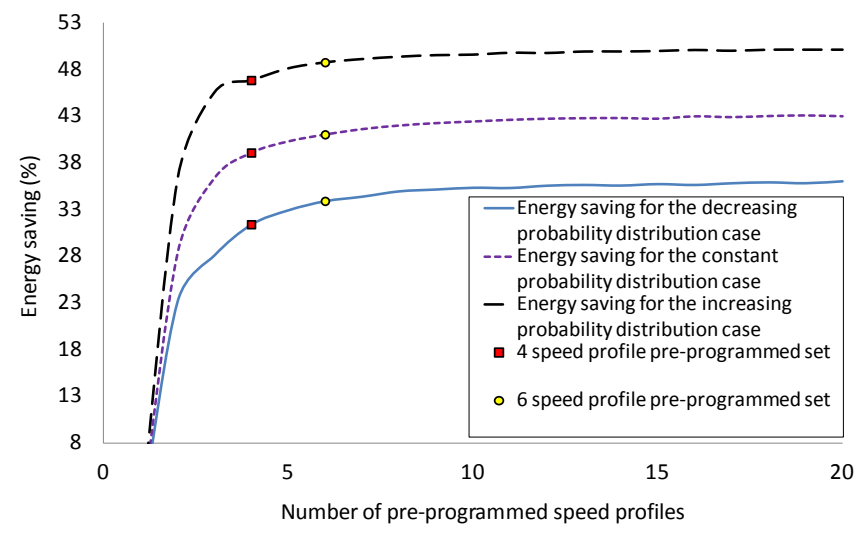

Fig. 13: Expected value of energy savings obtained for different preprogrammed set size compared with the pre-programmed of 1 speed profile

\section{CONCLUSIONS}

In this paper a new procedure to design energy efficient speed profiles to program in the signalling equipment of a metropolitan system has been proposed. The procedure takes into account the main uncertainties in the traffic operation: train load and delays in the line.

The proposed model is based on the calculation of the Pareto curve of the possible speed profiles which are robust against passenger load variations. Then, the set of speed profiles to be programmed in the signalling system is taken from the robust Pareto front by means of a PSO optimization algorithm, taking into account energy efficiency and delays distribution in the line.

Two algorithms for obtaining the robust Pareto front have been proposed and compared using a case study. The first model is a robust multi-objective optimization algorithm that makes use of a robust definition as a restriction. The second one is an alternative method based on the robustness of the solution to changes in their driving pattern. It has been shown that pattern-robustness requirement is more restrictive than definition-II of robustness. Moreover, the pattern-robustness requirement is more useful because it guarantees the comfort of the speed profile. Besides that, the alternative procedure has found more solutions than the standard robust optimization algorithm. For this reason, the pattern recognition gives to the designer more possible solutions to choose and, some of them, have lower energy consumption for a given running time.

The proposed selection model including train delays information has been compared with the traditional selection method that distributes speed profiles uniformly in time. The results show important energy savings, around 3 - 14\%. This model has also been used to study the energy benefits obtained from increasing the number of speed profiles in the preprogrammed set. The typical size of this set is 4 and the energy consumption can be reduced $3.5 \%$ by the inclusion of two extra speed profiles.

\section{REFERENCES}

[1] X. Feng, H. Zhang, Y. Ding, Z. Liu, H. Peng, and B. Xu, “A Review Study on Traction Energy Saving of Rail Transport," Discrete Dyn. Nat. Soc., vol. 2013, Sep. 2013.

[2] M. Abril, M. A. Salido, and F. Barber, "Distributed search in railway scheduling problems," Eng. Appl. Artif. Intell., vol. 21, no. 5, pp. 744755, Aug. 2008.

[3] X. Feng, H. Liu, H. Zhang, B. Wang, and Q. Sun, "Rational Formations of a Metro Train Improve Its Efficiencies of Both Traction Energy Utilization and Passenger Transport," Math. Probl. Eng., vol. 2013, Sep. 2013.

[4] X. Yang, B. Ning, X. Li, and T. Tang, "A Two-Objective Timetable Optimization Model in Subway Systems," IEEE Trans. Intell. Transp. Syst., vol. Early Access Online, 2014.

[5] M. Peña-Alcaraz, A. Fernandez, A. P. Cucala, A. Ramos, and R. R. Pecharromán, "Optimal underground timetable design based on power flow for maximizing the use of regenerative-braking energy," Proc. Inst. Mech. Eng. Part F J. Rail Rapid Transit, Dec. 2011.

[6] X. Yang, X. Li, Z. Gao, H. Wang, and T. Tang, "A Cooperative Scheduling Model for Timetable Optimization in Subway Systems," IEEE Trans. Intell. Transp. Syst., vol. 14, no. 1, pp. 438-447, Mar. 2013.

[7] J.-W. Sheu and W.-S. Lin, "Energy-Saving Automatic Train Regulation Using Dual Heuristic Programming,” IEEE Trans. Veh. Technol., vol. 61, no. 4, pp. 1503-1514, May 2012.

[8] W.-S. Lin and J.-W. Sheu, "Optimization of Train Regulation and Energy Usage of Metro Lines Using an Adaptive-Optimal-Control Algorithm,” IEEE Trans. Autom. Sci. Eng., vol. 8, no. 4, pp. 855-864, Oct. 2011.

[9] J.-W. Sheu and W.-S. Lin, "Automatic train regulation with energy saving using dual heuristic programming," IET Electr. Syst. Transp., vol. 1, no. 2, pp. 80-89, Jun. 2011.

[10] L.-M. Jia and X.-D. Zhang, "Distributed intelligent railway traffic control based on fuzzy decisionmaking," Fuzzy Sets Syst., vol. 62, no. 3, pp. 255-265, Mar. 1994.

[11] A. Fay, "A fuzzy knowledge-based system for railway traffic control," Eng. Appl. Artif. Intell., vol. 13, no. 6, pp. 719-729, Dec. 2000.

[12] M. Domínguez, A. Fernández-Cardador, A. P. Cucala, and R. R. Pecharromán, "Energy Savings in Metropolitan Railway Substations Through Regenerative Energy Recovery and Optimal Design of ATO Speed Profiles," IEEE Trans. Autom. Sci. Eng., vol. 9, no. 3, pp. 496504, 2012.

[13] M. C. Falvo, R. Lamedica, R. Bartoni, and G. Maranzano, "Energy management in metro-transit systems: An innovative proposal toward 
an integrated and sustainable urban mobility system including plug-in electric vehicles,” Electr. Power Syst. Res., vol. 81, no. 12, pp. 21272138, Dec. 2011.

[14] S. Lu, P. Weston, S. Hillmansen, H. B. Gooi, and C. Roberts, "Increasing the Regenerative Braking Energy for Railway Vehicles," IEEE Trans. Intell. Transp. Syst., vol. Early Access Online, 2014

[15] V. De Martinis and M. Gallo, "Models and Methods to Optimise Train Speed Profiles with and without Energy Recovery Systems: A Suburban Test Case," Procedia - Soc. Behav. Sci., vol. 87, pp. 222233, Oct. 2013.

[16] A. González-Gil, R. Palacin, P. Batty, and J. P. Powell, "A systems approach to reduce urban rail energy consumption," Energy Convers. Manag., vol. 80, pp. 509-524, Apr. 2014.

[17] S. Su, T. Tang, X. Li, and Z. Gao, "Optimization of Multitrain Operations in a Subway System,” IEEE Trans. Intell. Transp. Syst., vol. 15, no. 2, pp. 673-684, Apr. 2014.

[18] I. Araujo-Vargas, K. Cano-Pulido, J. Ramirez-Hernandez, N. Mondragon-Escamilla, C. Nicolas-Villalva, A. Villarruel-Parra, D. Cortes-Rodriguez, and F. Perez-Pinal, “A Single DC-Source, SevenLevel Inverter for Utility Equipment of Railway, Power-Land Substations,” IEEE Trans. Ind. Appl., vol. Early Access Online, 2014.

[19] A. Albrecht, P. Howlett, P. Pudney, and X. Vu, "Optimal train control: Analysis of a new local optimization principle," in American Control Conference (ACC), 2011, pp. 1928-1933.

[20] R. Franke, P. Terwiesch, and M. Meyer, "An algorithm for the optimal control of the driving of trains," in Proceedings of the 39th IEEE Conference on Decision and Control, 2000, vol. 3, pp. 21232128 vol.3.

[21] Q. Gu, T. Tang, F. Cao, and Y. Song, “Energy-Efficient Train Operation in Urban Rail Transit Using Real-Time Traffic Information,” IEEE Trans. Intell. Transp. Syst., vol. 15, no. 3, pp. 1216-1233, Jun. 2014.

[22] E. Khmelnitsky, "On an optimal control problem of train operation," IEEE Trans. Autom. Control, vol. 45, no. 7, pp. 1257-1266, 2000.

[23] H. Ko, T. Koseki, and M. Miyatake, "Application of dynamic programming to the optimization of the running profile of a train,” in Publication of: WIT Press, 2004.

[24] M. Miyatake and H. Ko, "Optimization of Train Speed Profile for Minimum Energy Consumption,” IEEJ Trans. Electr. Electron. Eng. vol. 5, no. 3, pp. 263-269, 2010.

[25] E. Rodrigo, S. Tapia, J. Mera, and M. Soler, "Optimizing Electric Rail Energy Consumption Using the Lagrange Multiplier Technique,” $J$. Transp. Eng., vol. 139, no. 3, pp. 321-329, 2013.

[26] S. Su, X. Li, T. Tang, and Z. Gao, “A Subway Train Timetable Optimization Approach Based on Energy-Efficient Operation Strategy,” IEEE Trans. Intell. Transp. Syst., vol. 14, no. 2, pp. 883893, 2013.

[27] Y. Wang, B. De Schutter, T. J. J. van den Boom, and B. Ning, "Optimal trajectory planning for trains - A pseudospectral method and a mixed integer linear programming approach,” Transp. Res. Part C Emerg. Technol., vol. 29, pp. 97-114, Apr. 2013.

[28] Y. V. Bocharnikov, A. M. Tobias, and C. Roberts, "Reduction of train and net energy consumption using genetic algorithms for Trajectory Optimisation," in IET Conference on Railway Traction Systems (RTS 2010), 2010, pp. 1-5.

[29] Y. V. Bocharnikov, A. M. Tobias, C. Roberts, S. Hillmansen, and C. J. Goodman, "Optimal driving strategy for traction energy saving on DC suburban railways," IET Electr. Power Appl., vol. 1, no. 5, pp. 675-682, 2007.

[30] A. P. Cucala, A. Fernández, C. Sicre, and M. Domínguez, "Fuzzy optimal schedule of high speed train operation to minimize energy consumption with uncertain delays and driver's behavioral response," Eng. Appl. Artif. Intell., vol. 25, no. 8, pp. 1548-1557, Dec. 2012.

[31] S. Lu, S. Hillmansen, T. K. Ho, and C. Roberts, "Single-Train Trajectory Optimization,” IEEE Trans. Intell. Transp. Syst., vol. 14, no. 2, pp. 743-750, 2013.

[32] C. Sicre, A. P. Cucala, and A. Fernández-Cardador, "Real time regulation of efficient driving of high speed trains based on a genetic algorithm and a fuzzy model of manual driving," Eng. Appl. Artif. Intell., vol. 29, pp. 79-92, 2014.

[33] C. Sicre, A. P. Cucala, A. Fernández, and P. Lukaszewicz, "Modeling and optimizing energy-efficient manual driving on high-speed lines," IEEJ Trans. Electr. Electron. Eng., vol. 7, no. 6, pp. 633-640, 2012.
[34] K. K. Wong and T. K. Ho, "Dynamic coast control of train movement with genetic algorithm," Intern J Syst Sci, vol. 35, no. 13-14, pp. 835846, Oct. 2004.

[35] K. K. Wong and T. K. Ho, "Coast control of train movement with genetic algorithm," in The 2003 Congress on Evolutionary Computation, 2003. CEC '03, 2003, vol. 2, pp. 1280-1287 Vol.2.

[36] L. Yang, K. Li, Z. Gao, and X. Li, "Optimizing trains movement on a railway network,” Omega, vol. 40, no. 5, pp. 619-633, Oct. 2012.

[37] X. Li and H. K. Lo, "An energy-efficient scheduling and speed control approach for metro rail operations,” Transp. Res. Part B Methodol., vol. 64, pp. 73-89, Jun. 2014.

[38] H.-J. Chuang, C.-S. Chen, C.-H. Lin, C.-H. Hsieh, and C.-Y. Ho, "Design of Optimal Coasting Speed for MRT Systems Using ANN Models,” IEEE Trans. Ind. Appl., vol. 45, no. 6, pp. 2090-2097, 2009.

[39] H.-J. Chuang, C.-S. Chen, C.-H. Lin, C.-H. Hsieh, and C.-Y. Ho, "Design of optimal coasting speed for saving social cost in Mass Rapid Transit systems," in Third International Conference on Electric Utility Deregulation and Restructuring and Power Technologies, 2008. DRPT 2008, 2008, pp. 2833-2839.

[40] S. Acikbas and M. T. Soylemez, "Coasting point optimisation for mass rail transit lines using artificial neural networks and genetic algorithms," IET Electr. Power Appl., vol. 2, no. 3, pp. 172-182, 2008.

[41] Y.-G. Kim, C.-S. Jeon, S.-W. Kim, and T.-W. Park, “Operating speed pattern optimization of railway vehicles with differential evolution algorithm,” Int. J. Automot. Technol., vol. 14, no. 6, pp. 903-911, Dec. 2013.

[42] Y. Bai, T. K. Ho, B. Mao, Y. Ding, and S. Chen, "Energy-Efficient Locomotive Operation for Chinese Mainline Railways by Fuzzy Predictive Control,” IEEE Trans. Intell. Transp. Syst., vol. 15, no. 3, pp. 938-948, Jun. 2014.

[43] F. De Cuadra, A. Fernandez, J. de Juan, and M. a. Herrero, "Energysaving automatic optimisation of train speed commands using direct search techniques,” presented at the Computers in Railways V - Vol.1 Railway Systems and Management, 1996, vol. 1.

[44] K. K. Wong and T. K. Ho, "Coast control for mass rapid transit railways with searching methods," IEE Proc. - Electr. Power Appl., vol. 151, no. 3, p. 365, 2004

[45] X. Zhu, R. Zhang, W. Dai, Z. Zhang, and J. Li, "Performance and Safety Assessment of ATO Systems in Urban Rail Transit Systems in China," J. Transp. Eng., vol. 139, no. 7, pp. 728-737, 2013.

[46] A. Fernandez, A. P. Cucala, B. Vitoriano, and F. de Cuadra, "Predictive Traffic Regulation for Metro Loop Lines Based on Quadratic Programming,” Proc. Inst. Mech. Eng. Part F J. Rail Rapid Transit, vol. 220, no. 2, pp. 79-89, Mar. 2006.

[47] M. Domínguez, A. Fernández, A. P. Cucala, and P. Lukaszewicz, "Optimal design of metro automatic train operation speed profiles for reducing energy consumption,” Proc. Inst. Mech. Eng. Part F J. Rail Rapid Transit, vol. 225, no. 5, pp. 463-474, Sep. 2011.

[48] "IEEE Standard for Communications-Based Train Control (CBTC) Performance and Functional Requirements,” IEEE Std 14741-1999, 1999.

[49] Q. Gu, X.-Y. Lu, and T. Tang, "Energy saving for automatic train control in moving block signaling system," in 2011 14th International IEEE Conference on Intelligent Transportation Systems (ITSC), 2011, pp. $1305-1310$.

[50] Y. Ding, Y. Bai, F. Liu, and B. Mao, "Simulation Algorithm for Energy-Efficient Train Control under Moving Block System,” in 2009 WRI World Congress on Computer Science and Information Engineering, 2009, vol. 5, pp. 498 -502.

[51] M. Domínguez, A. Fernández-Cardador, A. P. Cucala, T. Gonsalves, and A. Fernández-Rodríguez, "Multi objective particle swarm optimization algorithm for the design of efficient ATO speed profiles in metro lines,” Eng. Appl. Artif. Intell., vol. 29, pp. 43-53, Mar. 2014.

[52] B.-R. Ke, C.-L. Lin, and C.-C. Yang, "Optimisation of train energyefficient operation for mass rapid transit systems," IET Intell. Transp. Syst., vol. 6, no. 1, pp. 58 -66, Mar. 2012.

[53] W.-S. Lin and J.-W. Sheu, "Adaptive critic design of automatic train regulation of MRT system,” presented at the IEEE International Conference on Industrial Technology, 2008. ICIT 2008, pp. 1 -7.

[54] J. Kennedy and R. C. Eberhart, Swarm intelligence. San Francisco, CA, USA: Morgan Kaufmann Publishers Inc., 2001.

[55] K. Deb and H. Gupta, "Searching for Robust Pareto-Optimal Solutions in Multi-objective Optimization," in Evolutionary MultiCriterion Optimization, vol. 3410, C. A. Coello Coello, A. Hernández 
Aguirre, and E. Zitzler, Eds. Berlin, Heidelberg: Springer Berlin Heidelberg, 2005, pp. 150-164.

[56] C. R. Raquel and P. C. Naval,Jr., "An effective use of crowding distance in multiobjective particle swarm optimization," in

Proceedings of the 2005 conference on Genetic and evolutionary computation, New York, NY, USA, 2005, pp. 257-264.

[57] J. Kennedy and R. Eberhart, "Particle swarm optimization," in , IEEE International Conference on Neural Networks, 1995. Proceedings, 1995, vol. 4, pp. 1942-1948 vol.4.

[58] J. Kennedy and R. C. Eberhart, "A discrete binary version of the particle swarm algorithm," in , 1997 IEEE International Conference on Systems, Man, and Cybernetics, 1997. Computational Cybernetics and Simulation, 1997, vol. 5, pp. 4104-4108 vol.5. 